\title{
Cellular and Extracellular Siderophores of Aspergillus nidulans and Penicillium chrysogenum
}

\author{
GISELA CHARLANG, ${ }^{1}$ BRADFORD NG, ${ }^{1}$ NORMAN H. HOROWITZ, ${ }^{1 *}$ AND ROBERT M. HOROWITZ ${ }^{2}$ \\ Division of Biology, California Institute of Technology, Pasadena, California $91125,{ }^{1}$ and U.S. Department \\ of Agriculture, Fruit and Vegetable Chemistry Laboratory, Pasadena, California $91106^{2}$
}

\begin{abstract}
Aspergillus nidulans and Penicillium chrysogenum produce specific cellular siderophores in addition to the well-known siderophores of the culture medium. Since this was found previously in Neurospora crassa, it is probably generally true for filamentous ascomycetes. The cellular siderophore of $A$. nidulans is ferricrocin; that of $P$. chrysogenum is ferrichrome. A. nidulans also contains triacetylfusigen, a siderophore without apparent biological activity. Conidia of both species lose siderophores at high salt concentrations and become siderophore dependent. This has also been found in $N$. crassa, where lowering of the water activity has been shown to be the causal factor. We used an assay procedure based on this dependency to reexamine the extracellular siderophores of these species. During rapid mycelial growth, both $A$. nidulans and $P$. chrysogenum produced two highly active, unidentified siderophores which were later replaced by a less active or inactive product-coprogen in the case of $P$. chrysogenum and triacetylfusigen in the case of $A$. nidulans. $N$. crassa secreted coprogen only. Fungal siderophore metabolism is varied and complex.
\end{abstract}

Microorganisms elaborate a variety of lowmolecular-weight, high-affinity chelating agents that solubilize ferric iron in the environment and transport it into the cell (10). These compounds are known generically as "siderophores" (formerly "siderochromes"). Fungal siderophores are typically found in high concentrations in the filtrates of iron-deficient cultures, and it has generally been supposed that they are extracellular agents secreted in response to iron deficiency. In a study carried out for an unrelated purpose with Neurospora crassa, however, we found that exposure of conidia to solutions of low water activity $\left(a_{w}\right)$ causes them to lose a substance that is required for their germination (2). The substance was isolated and identified as the cyclic peptide ferricrocin (6), a known fungal siderophore (Fig. 1). Ferricrocin was found in the mycelia, as well as the conidia, of $N$. crassa grown on iron-sufficient media, but not in the culture filtrate. A structurally and functionally related compound, coprogen, had previously been shown to be present in iron-deficient culture filtrates (8). Ferricrocin and coprogen are equally active in promoting conidial germination at low $a_{w}$. The significance of the production of distinct cellular and extracellular siderophores is not yet clear.

To assess the generality of these findings, we performed experiments with Aspergillus nidulans and Penicillium chrysogenum. Extracellular siderophores had previously been identified in both species (12). Our study showed that specific cellular siderophores were also produced. Furthermore, as in $N$. crassa, the conidia of these species lost cellular siderophores at high salt concentrations and thereby became siderophore dependent. Each species could thus be used to assay siderophores. We used this assay to reexamine the production of extracellular siderophores by the two species. The findings revealed new facts of unusual interest.

\section{MATERIALS AND METHODS}

Strains. $P$. chrysogenum was obtained from the American Type Culture Collection, Rockville, Md. (ATCC 9480). A. nidulans was obtained from the Fungal Genetics Stock Center, Arcata, Calif. (FGSC 4, Glasgow wild type). $N$. crassa was wild type $74 \mathrm{~A}$, also from the Fungal Genetics Stock Center.

Media. Aspergillus and Penicillium were maintained on slants of potato-dextrose agar (Difco Laboratories, Detroit, Mich.). The same medium, with added agar, was used in Fernbach flasks for the production of conidia. For measuring responses to siderophores with Aspergillus and Penicillium, we used Vogel medium N (11) with the following additions per liter of water: sucrose, $20 \mathrm{~g}$; yeast extract, $5 \mathrm{~g}$; casein hydrolysate, $2.5 \mathrm{~g} ; \mathrm{MgSO}_{4} \cdot 7 \mathrm{H}_{2} \mathrm{O}, 5 \mathrm{~g}$; and $\mathrm{NaCl}, 194.35$ g. When Neurospora was used, casein hydrolysate was omitted and the $\mathrm{NaCl}$ was reduced to $103.1 \mathrm{~g}$. For isolating cellular siderophores, cultures were grown in medium N containing $2 \%$ sucrose with no other additions. For isolating extracellular siderophores, the Fedeficient "Normalmedium" of Kappner et al. (7) was used.

Measurement of growth. Mycelia were harvested on a Büchner funnel, using Whatman no. 4 paper. 


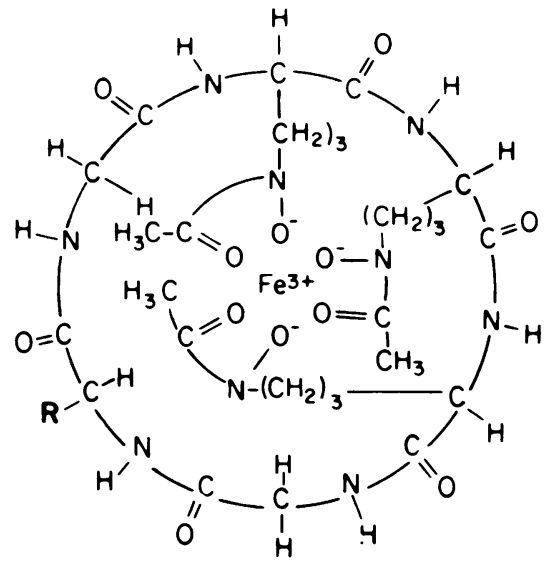

(a)

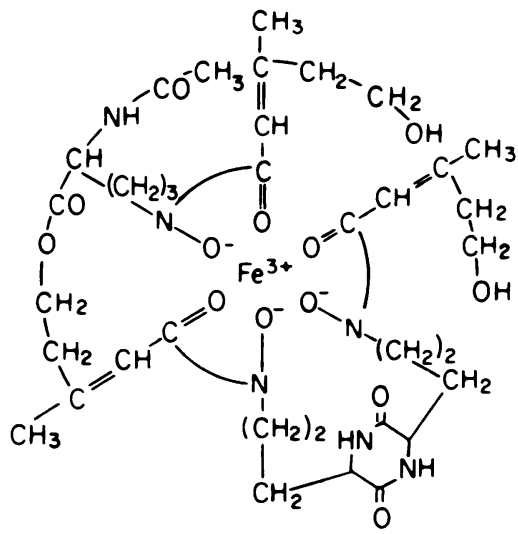

(b)

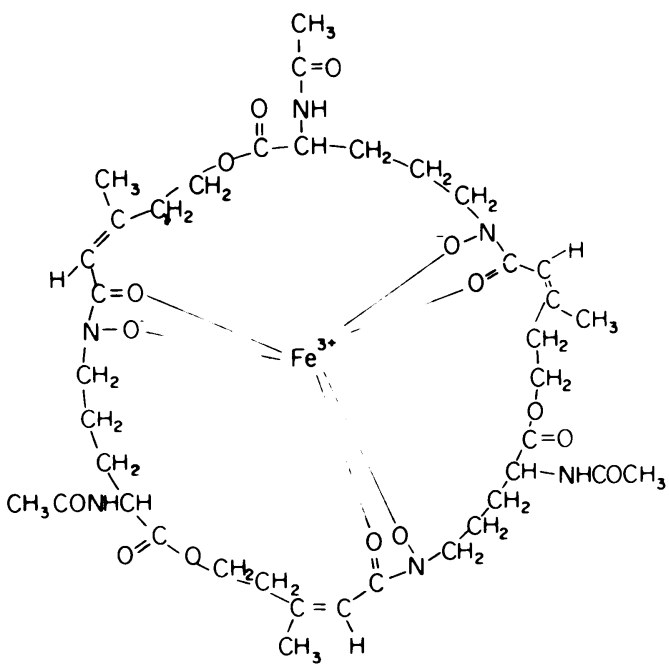

(C)
Excess moisture was removed by pressing mycelia between paper towels, and the mycelial pads were dried to constant weight at 70 to $80^{\circ} \mathrm{C}$.

Isolation of cellular siderophores. Three-dayold mycelia were washed, blended in an equal weight of water, and extracted for $1 \mathrm{~h}$ at $90^{\circ} \mathrm{C}$. The filtrate was cooled, adjusted to $\mathrm{pH} 5$ with $\mathrm{HCl}$, allowed to stand overnight at $4^{\circ} \mathrm{C}$, and filtered through Celite. The filtrate was brought to $\mathrm{pH} 6.5$ to 7 and taken to dryness. The dried extract was taken up in a minimal volume of water and poured into 20 volumes of cold 95\% ethanol, with stirring. The precipitate was removed by centrifigation and discarded. The supernatant was concentrated to dryness, taken up in a small volume of water, and poured into a column ( 20 by 50 cm) of Amberlite XAD-2 (Rohm \& Haas Co., Philadelphia, $\mathrm{Pa}$.). The column was washed with 20 bed volumes of water and eluted with 2 bed volumes of 1:1 acetone-water. The eluate was concentrated to a small volume and lyophilized. It was then dissolved in a small volume of water and layered on a column (40 by $1 \mathrm{~cm}$ ) of Bio-Gel P-2 (50 to 100 mesh; Bio-Rad Laboratories, Richmond, Calif.); the siderophore was eluted with water. The eluate was dried, dissolved in 2:1:1 chloroform-benzyl alcohol-methanol, and chromatographed on a column (20 by $2 \mathrm{~cm}$ ) of Silica Gel 60 (E. Merck AG, Darmstadt, Federal Republic of Germany), using the same solvent. The siderophore was transferred into water and lyophilized.

To obtain siderophores from conidia, conidia were harvested from cultures on potato-dextrose agar into $0.1 \%$ Tween 80 with the aid of a large wire loop. After filtering and washing the cells, the suspension was saturated with chloroform and shaken for 1 to $2 \mathrm{~h}$ at $30^{\circ} \mathrm{C}$. The conidia were removed by centrifugation, and the aqueous phase was separated by pipette, concentrated, and chromatographed on Amberlite XAD2 as above. Subsequent steps were as described above.

Isolation of extracellular siderophores. Fiveday cultures were filtered, and excess $\mathrm{FeCl}_{3}$ was added to the filtrate to convert desferrisiderophores to the ferric forms. Any precipitate was removed by centrifugation. The supernatant was poured onto a column of Amberlite XAD-2 (15 by $6 \mathrm{~cm}$ per 3 liters). The column was washed with water and eluted with acetone-water as above. The eluate was taken to dryness, redissolved in a small volume of water, and chromatographed on a column (15 by $1.5 \mathrm{~cm}$ ) of Cellex CM (Bio-Rad). Neutral siderophores were eluted with water, and cationic ones were eluted with pyridine-acetate buffer (acetic acid-pyridine-water, 10:14:930 by volume). The fractions were concentrated and lyophilized. An alternative procedure for neutral siderophores was, after Amberlite XAD-2 chromatography, dissolution of the eluate in 2:1:1 chloroform-benzyl alcohol-methanol followed by chromatography on Silica Gel 60 as above.

Analytical methods. ${ }^{13} \mathrm{C}$ nuclear magnetic resonance spectroscopy was performed with the same instrument and procedures as were used previously (6).

Fig. 1. Structures of $A$. nidulans and $P$. chrysogenum siderophores. (a) Ferrichrome, $R=H$; ferricrocin, $\mathrm{R}=\mathrm{CH}_{2} \mathrm{OH}$. (b) Coprogen. (c) $N, N^{\prime}, N^{\prime \prime}$.Triacetylfusigen. 
Thin-layer chromatography was ascending on silica gel sheets (Eastman Kodak Co., Rochester, N.Y.). Paper electrophoresis was carried out at $200 \mathrm{~V}$ using pyridine-acetate buffer, $\mathrm{pH}$ 5.2, and Whatman 3MM paper; runs lasted 2 to $3 \mathrm{~h}$. Amino acid analyses were performed as previously described (6).

\section{RESULTS}

$A$. nidulans and $P$. chrysogenum at low $a_{w}$. It has been shown in Neurospora that conidial germination is progressively inhibited as the water activity of the medium is reduced below $a_{w}=0.98$ (2). The degree of inhibition is a function of cell density, dense conidial suspensions being less inhibited than dilute ones. This "population effect" $(1,2)$ has been shown to be due to the reversible loss of a germination-essential substance from the cells; since dense suspensions produce a higher concentration of the substance than dilute ones, they germinate faster. It has been demonstrated that if a dense suspension is filtered to remove the cells and the filtrate is reinoculated with a dilute suspension, the latter germinates as if it were a dense suspension. The essential factor has been identified as ferricrocin (6). Addition of ferricrocin or certain other siderophores accelerates the germination of dilute suspensions of conidia in media of low $a_{w}$. This effect is the basis for a sensitive assay for siderophores in which, for convenience, growth of the cultures, rather than germination time, is measured $(2,6)$.

$A$. nidulans and $P$. chrysogenum were more resistant to low $a_{w}$ than $N$. crassa is. Whereas in Neurospora germination of dilute suspensions is strongly inhibited at $a_{w}=0.93$, the equivalent inhibition in Aspergillus and Penicillium occurred at $a_{u}=0.87$. Nevertheless, both of the latter species showed the characteristic population effect, and in both species the disadvantage of dilute suspensions was eliminated if an appropriate siderophore was added to the solution. These effects are shown in Table 1. The population effect is shown in the first two lines of each experiment (no added siderophore). The results of adding siderophores to a dilute suspension then follow. Growth weights in the table are normalized to equal numbers of conidia in order to give an unbiased comparison between high-density and low-density cultures. Thus, in $A$. nidulans experiment 1 , the first line shows growth in a 50-ml culture inoculated with $10^{3}$ conidia per $\mathrm{ml}$, whereas the second line shows the same for a $50-\mathrm{ml}$ culture inoculated with $10^{2}$ conidia per ml, multiplied by 10 . The same correction is applied to the subsequent values-i.e., in each experiment, dry weights are normalized to the number of conidia contained in the high-conidial-count control. The result is to remove the bias inherent in starting the cultures with unequal numbers of cells while preserving the effect of cell density.

We conclude from Table 1 that, as in $N$. crassa, loss of siderophores occurs at low $a_{w}$ in these species and is a major cause of the germination delay.

$\mathrm{NaCl}$ was used to lower $a_{u}$ in all of the experiments reported here. Proof that the effects described above were caused by low $a_{w}$ per se and not ion toxicity would require demonstration of the same phenomena in the presence of a nonelectrolyte as the $a_{u}$-lowering agent. This demonstration was possible with $N$. crassa (2), but not with $A$. nidulans and $P$. chrysogenum, owing, we believe, to side effects-such as high viscosity-associated with the large concentrations of glycerol or glucose required to bring $a_{u}$ to the levels that are effective with the latter species. In view of the results obtained with $\mathrm{NaCl}$, it is very likely that the same mechanism is at work in all three species, but the argument of this paper is independent of this assumption. The essential fact is that $A$. nidulans and $P$. chrysogenum could be made siderophore dependent by the described treatment.

Extracellular siderophores of $A$. nidulans and $P$. chrysogenum. Identification of the major siderophores secreted by various Aspergillus and Penicillium species was originally carried out by Zähner et al. (12). They found the principal siderophores in the media of $A$. nidulans and $P$. chrysogenum to be ferricrocin and coprogen, respectively. In addition to ferricrocin, $A$. nidulans produced a significant quantity of ferrirhodin.

Our study of these species confirmed the above finding for $P$. chrysogenum grown for 6 days or longer on the medium described by Kappner et al. (7) containing asparagine as the N source (see Materials and Methods). Under these conditions, coprogen was the predominant siderophore in the medium. In younger cultures, however, the major siderophore was an unidentified cationic compound which we call P- $\alpha$. P$\alpha$ was far more active for $P$. chrysogenum in the low- $a_{w}$ test than was coprogen (Table 1). Another unidentified cationic siderophore in young cultures was P- $\beta$. P- $\beta$ had almost the same biological activity as $\mathrm{P}-\alpha$. A third, less active cation, P- $\gamma$, was found in small amounts. P- $\alpha, P-\beta$, and $\mathrm{P}-\gamma$ essentially disappeared from the media of older cultures (Fig. 2). The identities of P- $\alpha$ and $P-\beta$ are under investigation.

If, instead of asparagine, glutamic acid plus urea was used as the $\mathrm{N}$ source for $P$. chrysogenum (7), the changes seen in Fig. 2 were greatly delayed (data not shown). Coprogen under these conditions did not appear in the 
TABLE 1. Growth responses to siderophores at low $a_{w}{ }^{a}$

\begin{tabular}{|c|c|c|c|c|c|}
\hline Species & Expt no. & $\begin{array}{c}\text { Log inoculum } \\
\text { (conidia/ml) }\end{array}$ & Addition & (concn in $\mu \mathrm{g} / \mathrm{ml}$ ) & $\begin{array}{c}\text { Growth } \\
\text { (mg dry wt) }\end{array}$ \\
\hline \multirow[t]{3}{*}{ A. nidulans } & 1 & $\begin{array}{l}3 \\
2 \\
2 \\
2 \\
2 \\
2\end{array}$ & $\begin{array}{l}\text { Ferricrocin } \\
\text { Ferrichrome } \\
\text { Ferrichrysin } \\
\text { Coprogen }\end{array}$ & $\begin{array}{l}(0.1) \\
(0.1) \\
(0.1) \\
(0.1)\end{array}$ & $\begin{array}{r}178 \\
7.6 \\
84.4 \\
42.8 \\
46.0 \\
2.4\end{array}$ \\
\hline & 2 & $\begin{array}{l}3 \\
2 \\
2 \\
2 \\
2 \\
2 \\
2 \\
2\end{array}$ & $\begin{array}{l}\text { Ferricrocin } \\
\text { Ferricrocin } \\
\text { A- } \alpha \\
\text { A- } \beta \\
\text { P- } \alpha \\
\text { P- } \beta\end{array}$ & $\begin{array}{l}(0.2) \\
(0.4) \\
(0.2) \\
(0.2) \\
(0.2) \\
(0.2)\end{array}$ & $\begin{array}{r}139 \\
\sim 1 \\
111 \\
215 \\
666 \\
630 \\
635 \\
646\end{array}$ \\
\hline & 3 & $\begin{array}{l}3 \\
2 \\
2 \\
2\end{array}$ & $\begin{array}{l}\text { Ferricrocin } \\
\text { Triacetylfusigen }\end{array}$ & $\begin{array}{l}(0.2) \\
(0.2)\end{array}$ & $\begin{array}{r}197 \\
45 \\
312 \\
48\end{array}$ \\
\hline \multirow[t]{2}{*}{ P. chrysogenum } & 1 & $\begin{array}{l}4 \\
3 \\
3 \\
3 \\
3 \\
3 \\
3\end{array}$ & $\begin{array}{l}\text { Ferricrocin } \\
\text { Ferrichrysin } \\
\text { Ferrichrome } \\
\text { Coprogen }\end{array}$ & $\begin{array}{l}(0.05) \\
(0.05) \\
(0.05) \\
(0.05)\end{array}$ & $\begin{array}{l}94.7 \\
15.4 \\
25 \\
22 \\
33 \\
124\end{array}$ \\
\hline & 2 & $\begin{array}{l}4 \\
3 \\
3 \\
3 \\
3 \\
3 \\
3 \\
3\end{array}$ & $\begin{array}{l}\text { Coprogen } \\
\text { P- } \alpha \\
\text { P- } \beta \\
\text { A- } \alpha \\
\text { A- } \beta \\
\text { Triacetylfusigen }\end{array}$ & $\begin{array}{l}(0.2) \\
(0.2) \\
(0.2) \\
(0.2) \\
(0.2) \\
(0.2)\end{array}$ & $\begin{array}{r}116 \\
\sim 1 \\
131 \\
2,190 \\
2,115 \\
2,106 \\
2,122 \\
\sim 1\end{array}$ \\
\hline
\end{tabular}

${ }^{a}$ Incubations were in $50 \mathrm{ml}$ of medium $\mathrm{N}$ with additions as listed in the text. Incubations were for 8 days at $30^{\circ} \mathrm{C}$ in the case of $A$. nidulans and 7 days at 25 to $26^{\circ} \mathrm{C}$ for $P$. chrysogenum with shaking. Three drops (40 mg) of Tween 80 was added to each $50 \mathrm{ml}$ of medium. $a_{w} \simeq 0.87$.

${ }^{b}$ Dry weights are normalized to the number of conidia used per $50 \mathrm{ml}$ in the high-conidial-count control; see the text for details.

culture filtrates until after 3 weeks of incubation. This delay was correlated with slower growth in the glutamic acid-urea medium.

In the case of $A$. nidulans, we were unable to detect either ferricrocin or ferrirhodin, the reported principal siderophores of the culture medium (12). Instead, we found a siderophore ("X") whose chromatographic properties differed markedly from those of ferricrocin and ferrirhodin (Table 2) and whose $\mathrm{HI}$ hydrolysate contained ornithine, but no other amino acids. " $X$ " was isolated and identified as the cyclic triester $N, N^{\prime}, N^{\prime \prime}$-triacetylfusigen, also called $N, N^{\prime}, N^{\prime \prime}$ triacetylfusarinine-C $(3,4,9)$. The identification was based on thin-layer chromatography and nuclear magnetic resonance spectroscopy (Table 3).
Biological tests of $N, N^{\prime}, N^{\prime \prime}$-triacetylfusigen gave the surprising result that this substance was inactive for both species used in this study, including the one that produces it (Table 1). It was also inactive for Neurospora. Two very active siderophores were, however, found in the media of young (3-day) Aspergillus cultures. These disappeared from older cultures. The properties of the two active compounds were similar to those of P- $\alpha$ and P- $\beta$, and they were designated $A-\alpha$ and $A-\beta$, respectively. These substances are under investigation.

Cellular siderophores of $\boldsymbol{A}$. nidulans and $P$. chrysogenum. By "cellular siderophores" we mean siderophores located in or on cells. Our experiments with Neurospora had suggested that conidia contain siderophores intracellularly 
and also bound to the cell surface (6). The presence of cellular siderophores in A. nidulans and $P$. chrysogenum has already been indicated in the results displayed in Table 1 . To identify the substances, siderophores were isolated from mycelia and conidia by the procedures described in Materials and Methods. Identification was made by thin-layer chromatography and amino acid analysis of $\mathrm{HI}$ hydrolysates (6).

The mycelium of $A$. nidulans was found to contain ferricrocin and $N, N^{\prime}, N^{\prime \prime}$-triacetylfusigen in the approximate ratio 1:3.5. Conidia contained ferricrocin only. The chromatographic behavior

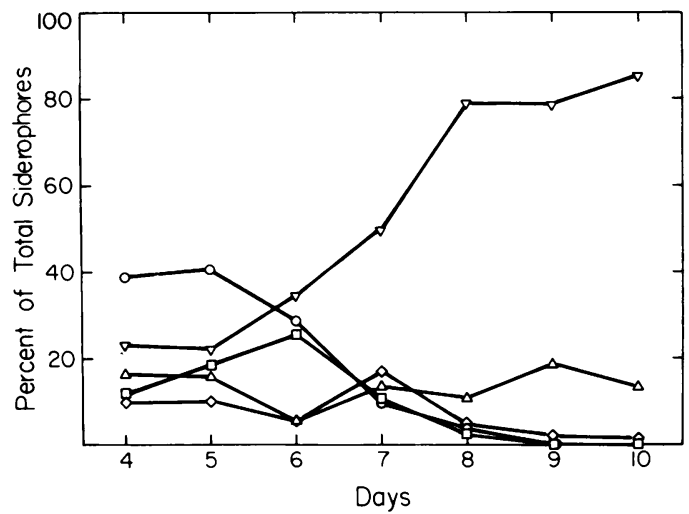

FIG. 2. Extracellular siderophores of P. chrysogenum as a function of age of culture. Asparagine was the $N$ source. Siderophores were separated by paper electrophoresis. Symbols: $\bigcirc, P-\alpha ; \nabla$, neutral siderophores (essentially all coprogen after day 5); $\triangle$, unknown, biologically inactive anions; $\square, P \cdot \beta ; \diamond$, $P-\gamma$. of the isolated compound, compared with authentic ferricrocin, is shown in Table 2. Analysis of a 4-h HI hydrolysate showed ornithine, glycine, and serine in the ratio 3.05:2:0.9.

$P$. chrysogenum produced ferrichrome as the only detected cellular siderophore (Table 2). The HI hydrolysate showed ornithine and glycine in the ratio 1.08:1.

Amounts of siderophores produced. We compared the concentrations of cellular and extracellular siderophores in order to determine whether one class predominates. The concentrations vary with age and conditions of culture, but we need only order-of-magnitude estimates for our purpose. Table 4 shows the concentrations derived from the present study and from the earlier one with Neurospora (6). Cellular siderophore concentrations are given per liter of cell water, assuming $80 \%$ water in fresh mycelium. Extracellular concentrations are with reference to the medium. It is evident that the two classes of siderophores are present in comparable concentrations in cultures of similar age. Since the volume of the medium exceeded that of the cell water by several orders of magnitude, however, it also follows that the total production of extracellular siderophores greatly exceeds that of cellular siderophores.

\section{DISCUSSION}

The results described above support the generalization that ascomycetous fungi produce specific cellular siderophores that are distinct from those secreted into the medium. The cellular siderophores are produced in concentrations

TABLE 2. $R_{f}$ values of siderophores in five solvents

\begin{tabular}{|c|c|c|c|c|c|}
\hline \multirow{2}{*}{ Siderophore } & \multicolumn{5}{|c|}{$R_{f}$ in solvent: ${ }^{a}$} \\
\hline & 1 & 2 & 3 & 4 & 5 \\
\hline Ferrichrome & 0.55 & 0.30 & 0.07 & 0.31 & 0.19 \\
\hline Ferricrocin & 0.55 & 0.25 & 0.04 & 0.39 & 0.20 \\
\hline Coprogen & 0.56 & 0.18 & 0.32 & 0.54 & 0.44 \\
\hline Ferrirhodin & 0.70 & 0.41 & 0.18 & 0.63 & 0.58 \\
\hline$N, N^{\prime}, N^{\prime \prime}-$ Triacetylfusigen & 0.81 & 0.55 & 0.42 & 0.61 & 0.64 \\
\hline \multicolumn{6}{|l|}{ From $A$. nidulans: } \\
\hline Cellular (ferricrocin) & 0.56 & 0.24 & 0.03 & 0.40 & 0.19 \\
\hline X (triacetylfusigen) & 0.83 & 0.55 & 0.42 & 0.61 & 0.66 \\
\hline A- $\alpha$ & $0.16^{b}$ & $0.08^{b}$ & 0.03 & 0.08 & 0.02 \\
\hline$A-\beta$ & 0.00 & 0.00 & 0.00 & 0.03 & 0.02 \\
\hline \multicolumn{6}{|l|}{ From $P$. chrysogenum: } \\
\hline Cellular (ferrichrome) & 0.56 & 0.30 & 0.07 & 0.32 & 0.19 \\
\hline Extracellular (coprogen) & 0.57 & 0.18 & 0.31 & 0.55 & 0.45 \\
\hline P- $\alpha$ & $0.16^{b}$ & $0.12^{b}$ & 0.04 & 0.03 & 0.02 \\
\hline P- $\beta$ & 0.00 & 0.00 & 0.00 & 0.03 & 0.02 \\
\hline
\end{tabular}

${ }^{a}$ Thin-layer chromatography (Eastman silica gel sheets) in the following solvents: (1) chloroform-benzyl alcohol-methanol, 2:1:1 by volume; (2) chloroform-propanol-methanol-water, 18:7:2:1 by volume; (3) butanolpropanol-methanol, 1:1:1 by volume; (4) butanol-ethanol-water, 2:1:2 by volume; and (5) butanol-acetic acidwater, $6: 1: 1$ by volume.

${ }^{b}$ Streaked. 
comparable to those observed for extracellular siderophores, but in much smaller absolute quantities. The impression sometimes gained from the literature that fungal siderophores are produced only to perform a rescue operation during times of iron starvation is clearly erroneous. Although the rescue function is an important one, our results show that these substances are synthesized and function under conditions of adequate iron nutrition as well.

In the three species we have studied, cellular siderophores have a role in conidial germination. Since conidia are dormant cells that require a

TABLE $3 .{ }^{13} \mathrm{C}$ chemical shifts of $N, N^{\prime}, N^{\prime \prime}$. triacetyldesferrifusigen and desferri- $\mathrm{X}^{a}$

\begin{tabular}{|c|c|c|}
\hline Carbon & $\begin{array}{l}\text { Triacetyldesfer- } \\
\text { rifusigen }\end{array}$ & Desferri-X \\
\hline $\mathrm{CH}_{3} \mathrm{CONH}-$ & $22.9 \mathrm{q}$ & 22.9 \\
\hline$-\mathrm{CH}_{2} \mathrm{CH}_{2} \mathrm{CH}_{2} \mathrm{CH}<$ & $52.5 \mathrm{~d}$ & 52.5 \\
\hline$-\mathrm{CH}_{2} \mathrm{CH}_{2} \mathrm{CH}_{2} \mathrm{CH}<$ & $29.3 \mathrm{tr}$ & 29.3 \\
\hline$-\mathrm{CH}_{2} \mathrm{CH}_{2} \mathrm{CH}_{2} \mathrm{CH}<$ & $23.3 \mathrm{tr}$ & 23.3 \\
\hline$-\mathrm{CH}_{2} \mathrm{CH}_{2} \mathrm{CH}_{2} \mathrm{CH}<$ & $48.1 \mathrm{tr}$ & 48.1 \\
\hline$=\mathrm{CH}-$ & $118.4 \mathrm{~d}$ & 118.4 \\
\hline$>\mathrm{C}=$ & $149.1 \mathrm{~s}$ & 149.1 \\
\hline $\mathrm{CH}_{3}-\mathrm{C}=$ & $24.4 \mathrm{q}$ & 24.4 \\
\hline$-\mathrm{CH}_{2}-\mathrm{C}=$ & $32.4 \mathrm{tr}$ & 32.4 \\
\hline$-\mathrm{O}-\mathrm{CH}_{2}-$ & $62.9 \mathrm{tr}$ & 62.9 \\
\hline $\mathrm{O}=\mathrm{C}-\mathrm{O}$ & $170.9^{b}$ & 170.9 \\
\hline $\mathrm{O}=\mathrm{C}-\mathrm{NH}-$ & $172.0^{b}$ & 172.1 \\
\hline $\mathrm{O}=\mathrm{C}-\mathrm{NOH}-$ & 172.0 & 172.1 \\
\hline
\end{tabular}

${ }^{a}$ In $\mathrm{CDCl}_{3}$ at $46^{\circ} \mathrm{C}$; chemical shifts in parts per million downfield from tetramethylsilane; s, d, tr, and $q$ are multiplicities (single, double, triplet, and quartet, respectively) observed in single-frequency off-resonance decoupling.

${ }^{b}$ May be interchanged. large input of iron for germination, it is understandable that they are provided with a siderophore that remains in the cell rather than one that is secreted into the medium, where it might diffuse away before performing its function. It may be that this provisioning of conidia is the sole reason for the production of cellular siderophores. If so, there is a great waste of these compounds in fungal cultures, since only a small fraction of the total is found in conidia. It is an open question whether they have a mycelial function as well, such as transporting iron into the mitochondria.

The findings reported in this paper with respect to cellular siderophores largely parallel those made earlier with Neurospora. This is not the case for extracellular siderophores, however, where we find that Aspergillus and Penicillium depart widely from the Neurospora pattern. Whereas Neurospora produces coprogen from the beginning of growth, Aspergillus and Peni. cillium secrete several siderophores, and these change with time. The most interesting are the $\alpha$ 's and $\beta$ 's. These very active compounds are produced during the phase of rapid mycelial growth. In older cultures they are replaced by less active (coprogen) or inactive (triacetylfusigen) siderophores. Further study of these phenomena, including identification of the unknown substances, is obviously required. Similar results have recently been described by Emery for $\mathbf{F u}$ sarium roseum (5).

The accumulation of $N, N^{\prime}, N^{\prime \prime}$-triacetylfusigen by $A$. nidulans in large amounts is not understood. It does not seem to be a sign of senescence, since this inactive compound is also present in growing mycelia, although not-perhaps significantly-in conidia. Also not understood is the discrepancy between our strain of $A$. nidulans and that of Zähner et al. (12) with regard to their secreted siderophores. The discrepancy undoubtedly means that one of these strains should not be classified as $A$. nidulans. It suggests also

TABLE 4. Siderophore concentrations

\begin{tabular}{|c|c|c|c|c|}
\hline \multirow[t]{2}{*}{ Species } & \multirow{2}{*}{ Medium } & \multirow{2}{*}{$\begin{array}{l}\text { Age of culture } \\
\text { (days) }\end{array}$} & \multicolumn{2}{|c|}{$\begin{array}{l}\text { Siderophore concn } \\
(\mathrm{mg} / \text { liter })^{a}\end{array}$} \\
\hline & & & Cellular & Extracellular \\
\hline N. crassa & $\begin{array}{c}\mathbf{N} \\
\text { Normalmedium }\end{array}$ & $\begin{array}{r}2 \\
5 \\
5 \\
11\end{array}$ & $\begin{array}{c}10-15 \\
200 \\
600\end{array}$ & $\begin{array}{l}300 \\
500\end{array}$ \\
\hline P. chrysogenum & $\stackrel{\mathbf{N}}{\text { N }}$ & 5 & 125 & \\
\hline A. nidulans & $\begin{array}{c}\text { Normalmedium } \\
\mathbf{N} \\
\text { Normalmedium }\end{array}$ & $\begin{array}{l}\mathbf{5} \\
\mathbf{5} \\
\mathbf{5}\end{array}$ & $\begin{array}{l}160 \\
125\end{array}$ & $\begin{array}{l}200-300 \\
200-300\end{array}$ \\
\hline
\end{tabular}

\footnotetext{
${ }^{a}$ Concentrations are with reference to cell water for cellular siderophores and medium for extracellular ones.
} 
that siderophore identity may prove to be a useful trait in fungal taxonomy.

\section{ACKNOWLEDGMENTS}

This research was supported by Public Health Service grant AI15739 from the National Institute of Allergy and Infectious Diseases and by Biomedical Research Support Grant SO7 RR07003 from the Division of Research Resources, National Institutes of Health.

We are greatly indebted to Thomas Emery for a sample of $N, N^{\prime}, N^{\prime \prime}$-triacetylfusarinine-C and to W. Keller-Schierlein for samples of ferrichrome and ferricrocin. We thank J. Roger Shelton for performing the amino acid analyses.

\section{LTERATURE CITED}

1. Charlang, G., and N. P. Williams. 1977. Germinationdefective mutant of Neurospora crassa that responds to siderophores. J. Bacteriol. 132:1042-1044.

2. Charlang, G. W., and N. H. Horowitz. 1971. Germination and growth of Neurospora at low water activites. Proc. Natl. Acad. Sci. U.S.A. 68:260-262.

3. Diekmann, H., and E. Krezdorn. 1975. Stoffwechselprodukte von Mikroorganismen. 150. Mitteilung. Ferricrocin, Triacetylfusigen und andere Sideramine aus Pilzen der Gattung Aspergillus, Gruppe Fumigatus. Arch. Microbiol. 106: 191-194.

4. Diekmann, H., and H. Zähner. 1967. Konstitution von
Fusigen und dessen Abbau zu $\Delta^{\prime 2}$-Anhydromevalonsäurelacton. Eur. J. Biochem. 3:213-218.

5. Emery, T. 1980. Malonichrome, a new iron chelate from Fusarium roseum. Biochim. Biophys. Acta 629:382390.

6. Horowitz, N. H., G. Charlang, G. Horn, and N. P. Williams. 1976. Isolation and identification of the conidial germination factor of Neurospora crassa. J. Bacteriol. 127:135-140.

7. Kappner, M., A. Hasenböhler, and H. Zähner. 1977. Stoffwechselprodukte von Mikroorganismen. 166. Mitteilung. Optimierung der desferri-Ferricrocinbildung bei Aspergillus viridi-nutans Ducker \& Thrower. Arch. Microbiol. 115:323-331.

8. Keller-Schierlein, W., and H. Diekmann. 1970. Stoffwechselprodukte von Mikroorganismen. 85. Mitteilung. Zur Konstitution des Coprogens. Helv. Chim. Acta 53: 2035-2044.

9. Moore, R. E., and T. Emery. 1976. N"-Acetylfusarinines: isolation, characterization, and properties. Biochemistry 15:2719-2723.

10. Neilands, J. B. (ed.). 1974. Microbial iron metabolism. Academic Press, Inc., New York.

11. Vogel, H. J. 1964. Distribution of lysine pathways among fungi: evolutionary implications. Am. Nat. 98:435-446.

12. Zähner, H., W. Keller-Schierlein, R. Hütter, K. HessLeisinger, and A. Deer. 1963. Stoffwechselprodukte von Microorganismen. Sideramine aus Aspergillaceen. Arch. Mikrobiol. 45:119-135. 